\section{Polyacrylamide Gel as a Matrix for the Delivery of a Layer or Coat of Other Molecules}

\section{BioTechniques 30:258-262 (February 2001)}

In this work, we demonstrate the potential of thin polyacrylamide gels as a material or matrix for the incorporation of other molecules to deposit or deliver a uniform layer or coat of the molecules for applications unrelated to electrophoresis. Polyacrylamide has been used as a matrix for entrapment of a number of molecules for different purposes $(7,9,12)$. For instance, entrapment of collagen in polyacrylamide granules for the purification of collagenases has been described (12). However, in our knowledge, there is no report of the use of cast gels as thin layers for a coating application or delivery of a molecule in a layer onto another surface. We suggest that polyacrylamide gels can be used for overlaying applications, either wet or in dried form. As an example, in the present work, we have shown that a polyacrylamide gel in the dried form can be used for fluorography of tritiated small molecules.

Tritium is a radioisotope that has applications in experiments requiring ${ }^{3} \mathrm{H}$ labeled precursors. Film detection of the low-energy $\beta$ particles from tritium decay is very inefficient by autoradiography because these particles do not penetrate the silver halide photographic emulsion on X-ray film sufficiently (15). So, for detection of tritium-containing molecules, the technique of fluorography (11) was developed (18), in which a scintillant or fluor such as 2,5diphenyloxazole (PPO) (3) is used. Upon application, the scintillants emit light when the $\beta$ particles from radioactive disintegration impinge on them. Thus, the multiple photons generated by the fluor, rather than the weak $\beta$ particles from ${ }^{3} \mathrm{H}$ disintegration them selves, cause activation of the photographic emulsion on X-ray films. Thus, for film detection, the energy released by radioactive molecules is converted to visible light by solid-state scintillation (1). For fluorography of thin supports such as paper chromatograms, thin layer chromatography (TLC) plates, or nitrocellulose/nylon filters, the methods developed involve the incorporation of a scintillant into the thin support by dipping into a solution of scintillant $(4,10,14)$ or in melted scintillant (4), or by including a scintillant in the preparation of a thin layer of slurry for TLC (11). Spray formulations of scintillants are also available commercially (NEN ${ }^{\mathrm{TM}}$ Life Science Products, Boston, MA, USA). For fluorography of ${ }^{3} \mathrm{H}$-labeled molecules in polyacrylamide gels, a method using PPO has been described (3) in which the gel is soaked in dimethyl sulfoxide (DMSO) to replace water and then in a solution of PPO in DMSO. The PPO gets incorporated into the gel; the gel is placed in water so that the incorporated PPO precipitates within the gel matrix. The gel is dried, and X-ray film is exposed to it.

Here, an alternative method for fluorography of tritium-containing small molecules on thin filters is described. This procedure, adapted from the method described above for fluorography of polyacrylamide gels, recruits the capacity of polyacrylamide gel to be efficiently embedded with a large amount of PPO and uses a polyacrylamide gel as a matrix for application of PPO to the filter. The protocol followed is outlined below.

A $15 \%$ polyacrylamide gel mixture was prepared using acrylamide, bisacrylamide, TEMED, ammonium persulfate, and water. No SDS or Tris buffer was added. The solution was mixed thoroughly to ensure uniform lattice formation (important for even PPO incorporation) and poured in a Hoefer vertical gel cast (Hoefer Scientific, San Francisco, CA, USA) using 0.5 -mm spacers. A $15 \%$ gel concentration was chosen because these gels are easy to handle and do not break easily. The gel was removed from the cast and processed for fluorography as described earlier (2,3). Briefly, the gel was placed in DMSO for $2 \times 30$ min with gentle agitation. The gel was transferred into a $20 \%$ (w/v) solution of PPO (Loba Chemie, Mumbai, India) in DMSO and kept for $2 \mathrm{~h}$ with gentle agitation. The PPO-embedded gel was kept under slowly running water for $1 \mathrm{~h}$.

For demonstration of this method, $60,300,900,1500$, and $2700 \mathrm{dpm} / \mathrm{mm}^{2}$ spots of tritiated thymidine (NEN Life
Science Products) were made on Whatman no. 1 filter paper (Whatman, Kent, UK). Dot blot filters were prepared in triplicate, one filter serving as the nonfluorographed control, one to be fluorographed by dipping in a solution of scintillant, and one to be processed according to the method described in this work. The diameter of each spot was $4.5 \mathrm{~mm}$. The disintegrations per minute were determined, taking into consideration the half-life of tritium, and also verified using the Packard TriCarb 2100TR liquid scintillation counter (Packard Instrument, Downers Grove, IL, USA).

One filter was dipped into a solution of PPO in toluene (20\% w/v) (17) and air dried. The second filter to be fluorographed was placed on a Whatman no. 3 filter paper. The PPO-embedded gel, as prepared above, was cut to a size a little larger than the filter $(0.5-1 \mathrm{~cm})$ on all sides, lightly dabbed with tissue to remove excess water, and then this was placed over the filter, the gel extending a little beyond the filter on all four sides. The assembly was covered with cling film, and the gel was dried over the filter for $1 \mathrm{~h}$ at $65^{\circ} \mathrm{C}$ in a Bio-Rad gel drier (Bio-Rad Laboratories, Hercules, CA, USA). Marking the position of the filter is not required because the PPO-embedded gel becomes transparent upon drying and the paper becomes completely visible through the gel. The cling film was removed, and X-ray film was exposed to this filter over which a layer of PPO had been applied using a polyacrylamide gel. Thus, a PPO-embedded polyacrylamide (PEP) gel screen was placed over the filter before exposure. The film was exposed at $-70^{\circ} \mathrm{C}$. The filter that was not fluorographed and the dipped filter were placed, devoid of any wrap, next to the filter with PEP screen and autoradiographed on the same piece of X-ray film for the same time. The $\mathrm{X}$-ray film was developed for $5 \mathrm{~min}$ at $25^{\circ} \mathrm{C}-28^{\circ} \mathrm{C}$ and fixed. Kodak ${ }^{\circledR}$ X-Omat ${ }^{\circledR}$ AR film (Eastman Kodak, Rochester, NY, USA), which is highly sensitive to the blue light emitted from PPO (6) was used without preflashing (8). Kodak DA-163 developer (stock solution without dilution) was used.

The results are shown in Figure 1A. No signal was visible from the filter that was not fluorographed (not shown 
in figure). The results of the two fluorographed filters were comparable. With a 17-h exposure, $900 \mathrm{dpm} / \mathrm{mm}^{2}$ were visible on the film by both the dipping method described earlier and by our method. In Figure 1, an 8-days exposure is shown in which $60 \mathrm{dpm} / \mathrm{mm}^{2}$ is clearly visible. The signals from the different spots are seen with intensity of signal increasing according the amount of disintegrations per minute spotted. The sensitivities of the method described in this work and of the dipping method in our experimental setup are the same.

Thus, PPO-embedded polyacrylamide screens can be used for applications such as fluorography of paper chromatograms in which tritium-labeled small molecules on filters are to be detected $(5,13)$. The drying step renders the PPO layer transparent, so that light emitted can reach the X-ray film. Drying the PEP screen over the Whatman no. 1 filter brings the PPO within the range of the $\beta$ particles emitted from tritium, giving a sensitivity of 900

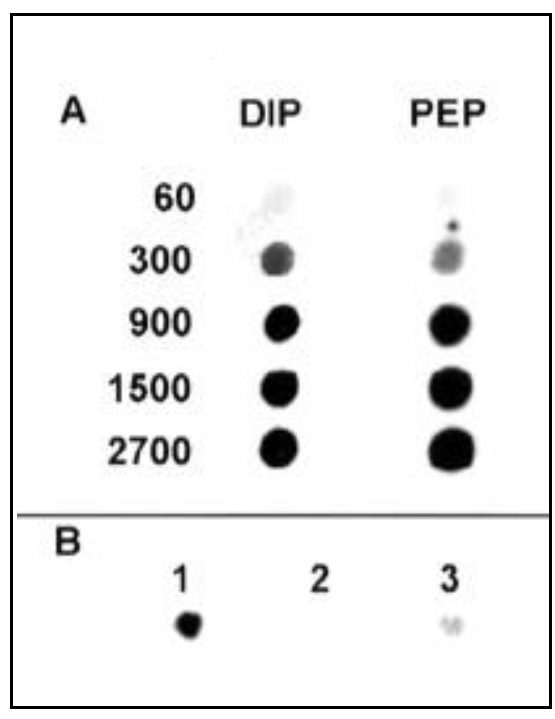

Figure 1. Use of a PPO-embedded polyacrylamide gel as a matrix to deliver a layer of the scintillant for fluorography of tritiated thymidine. (A) Increasing concentrations of ${ }^{3} \mathrm{H}$-thymidine were spotted on Whatman no. 1 filter paper. The numbers denote $\mathrm{dpm} / \mathrm{mm}^{2}$ spotted. DIP, filter fluorographed by dipping in a solution of PPO (17). PEP, duplicate filter fluorographed using PEP screen, a PPO-embedded polyacrylamide gel layered over the filter. An 8-days exposure is shown. (B) Use of PEP screen in the detection of DNase activity in cells. 1, DNase I (positive control); 2, negative control; 3 , hybridoma CC9C10 cell extract. $\mathrm{dpm} / \mathrm{mm}^{2}$ in an overnight exposure. In our method, we have found that a $20 \%$ PPO solution for incorporation into the gel to be placed on the filter gives a greater sensitivity than $22 \%$ PPO, which was the concentration in the original method for molecules inside gels (3).

The entire procedure takes about 5 $\mathrm{h}$, during which constant monitoring is not required. For a gel of $0.5-\mathrm{mm}$ thickness, this time can be reduced to $2 \mathrm{~h}$, as standardized as described elsewhere (2). Also, of practical significance, larger PEP screens (using a bigger gelcasting apparatus) can be prepared in advance and stored under water for long periods of time. At the time of use, the PPO-embedded gel is removed from water and cut to the desired size according to the filter. Once the PPOembedded gel is kept available, the method is very simple, involving only 1 $h$ of drying the gel over the filter.

With sprayed applications of scintillators, it may not be possible to achieve a perfectly even distribution of the scintillant over the filter; some areas of the filter may receive a little more or less spray than other areas. So, the scintillant could be more dense in some parts of the filter and less dense in others, which is also due to overlap of the sprayed areas in each of the applications. Uneven deposition of the fluor could obscure minute differences in the amount of radioactivity between bands or spots. In a detailed analysis of various factors that affect the tritium fluorographic process (14), it has been observed that it is almost impossible to achieve a uniform impregnation of scintillant by spraying. Difficulty in obtaining an even distribution of PPO has been experienced in pouring a solution over the filter (8). Also, dipping the filter in a scintillant solution within a few seconds can be tricky because, otherwise, rapid evaporation of the solvent may cause uneven distribution of the fluor. Substitution with less volatile solvents may give rise to formation of crystal deposits on the surface (14). PEP screen ensures a very uniform layer of PPO over the filter, once precision spacers and plates are used for pouring the gel and the gel is incubated in PPO solution until completely saturated. For additional exposures of the filter, reapplication of the scintillant is required when using a spray formulation. With PEP screen, as many different exposures as required can be obtained without any reapplication. Scintillant spray can leave a persistent odor in the exposure cassette. Also, unless the filter is covered in plastic wrap, this contaminating scintillant can be a problem for later experiments that require the cassette. PEP screen leaves no odor, and since PPO is contained within a dried polyacrylamide matrix, the cassette does not get contaminated with any scintillant. Also, PEP screen fluorography is devoid of the environmental hazards associated with an aerosol spray of a harmful compound. When a filter is dipped into a solution, there is the possibility that certain compounds on the filter may get displaced in certain solvents. For example, methanol- or ethanol-based solvents result in slightly diffuse spots (14). With PEP screen, this can be avoided, once excess moisture is carefully removed from the surface of the PPO-embedded gel. This 
method provides a viable alternative for fluorography of tritium-labeled small compounds when a spray application may not be available for any reason.

The following experiment was performed to demonstrate an actual application of the PEP screen, in which we show that PEP screen can be used in the detection of DNase activity in cell extracts. Here, we tested the B-cell hybridoma CC9C10 line. A plasmid was labeled with tritium by growing $E$. coli harboring the plasmid in LB medium containing tritiated thymidine overnight. The labeled plasmid DNA was isolated and incubated with DNase I (positive control), nuclease-free water (negative control), or with $7 \mu \mathrm{g}$ CC9C10 protein extract made by freeze-thawing the cells in $1 \times$ PBS. Undigested DNA in the reactions was precipitated with ethanol, and the reactions were centrifuged at $8000 \times g$ for $15 \mathrm{~min}$ at $4^{\circ} \mathrm{C}$. Ten microliters of each supernatant, containing free deoxynucleotides, if any, because of DNAse ac- tivity, were spotted on Whatman no. 1 paper, layered with PEP screen and Xray film exposed to the paper for 24 days. Figure 1B shows the detection of DNase activity in the CC9C10 extract. PEP screens could similarly be used for applications such as detection by paper chromatography of different dimeric forms of pyrimidines caused by UV irradiation (16).

The aim of evolving the technique described in this work is to reveal an example of a novel utility of the polyacrylamide gel-as a material for embedding another compound in a layer for an application unrelated to electrophoretic separation. Polyacrylamide can easily be cast into a thin layer that has useful physical and chemical properties, both in hydrated or dried forms. The fact that a polyacrylamide gel can be used as a carrier matrix for uniform coating or layering of a compound onto another surface, as demonstrated in our work, could lead to new kinds of applications of polyacrylamide gels other than in fluorography, in different areas of biochemistry or molecular biology.

\section{REFERENCES}

1.Ausubel, F.M, R. Brent, R.E. Kingston, J.G. Seidman, J.A. Smith, and K. Struhl. 1998. Current Protocols in Molecular Biology, Volume 3. John Wiley \& Sons, New York.

2.Bonner, W.M. 1983. Use of fluorography for sensitive isotope detection in polyacrylamide gel electrophoresis and related techniques. Methods Enzymol. 96:215-222.

3.Bonner, W.M. and R.A. Laskey. 1974. A film detection method for tritium labeled proteins and nucleic acids in polyacrylamide gels. Eur. J. Biochem. 46:83-88.

4.Bonner, W.M. and J.D. Stedman. 1978. Efficient fluorography of $3 \mathrm{H}$ and ${ }^{14} \mathrm{C}$ on thin layers. Anal. Biochem. 89:247-256.

5.Burton, K.S. and R.A. Consigli. 1996. Methylation of the polyomavirus major capsid protein VP1. Virus Res. 40:141-147.

6.Coligan, J.E., B.M. Dunn, H.L. Ploegh, D.W. Speicher, and P.T. Wingfield. 1997. Current Protocols in Protein Science. John Wiley \& Sons, New York.

7.Igloi, G.L. 1999. Automated detection of point mutations by electrophoresis in peptide nucleic acid-containing gels. BioTechniques 27:798-804.

8.Laskey, R.A. and A.D. Mills. 1975. Quantitative film detection of ${ }^{3} \mathrm{H}$ and ${ }^{14} \mathrm{C}$ in polyacrylamide by fluorography. Eur. J. Biochem. 56:335-341

9.Lewis, W.S. and S.M. Schuster. 1990. Quantitation of immobilized proteins. J. Biochem. Biophys. Methods 21:129-144.
10.Lucher, L.A. and T. Lego. 1989. Use of the water-soluble fluor sodium salicylate for fluorographic detection of tritium in thin-layer chromatograms and nitrocellulose filters. Anal. Biochem. 178:327-330.

11.Luthi, U. and P.G. Waser. 1965. Low-tem perature fluorography induced by tritium-labeled compounds on thin-layer chromatograms. Nature 205:1190-1191.

12.Nagai, Y. and H. Hori. 1972. Entrapment of collagen in a polyacrylamide matrix and its application in the purification of animal collagenases. Biochim. Biophys. Acta 263:564573.

13.Nakada, H., M. Inoue, Y. Numata, N. Tanaka, I. Funakoshi, S. Fukui, and I. Yamashina. 1992. Cancer-associated glycoproteins defined by a monoclonal antibody, MLS 128, recognizing the $\mathrm{Tn}$ antigen. Biochem. Biophys. Res. Commun. 187:217-224.

14.Randerath, K. 1970. An evaluation of film detection methods for weak $\beta$-emitters, particularly tritium. Anal. Biochem. 34:188-205.

15.Sambrook, J., E.F. Fritsch, and T. Maniatis. 1989. Molecular Cloning:A Laboratory Manual, 2nd edition. CSH Laboratory Press, Cold Spring Harbor, NY.

16.Setlow, R.B. 1966. Cyclobutane-type pyrimidine dimers in polynucleotides. Science 153:379-386.

17.Southern, E.M. 1975. Detection of specific sequences among DNA fragments separated by gel electrophoresis. J. Mol. Biol. 98:503517.

18. Wilson, A.T. 1958. Tritium and paper chromatography. Nature 182:524.

This work was supported by the Department of Biotechnology, Government of India. We wish to thank Dr. G.C. Mishra, Director, National Center for Cell Science (NCCS), Pune, for his encouragement and also all those at NCCS from whom help was received during the course of this work. Address correspondence to Dr. Manisha Deshpande, National Center for Cell Science, NCCS Complex, Ganeshkhind, Pune - 411007, India.e-mail:manisha_desh@hotmail.com

Received 7 February 2000; accepted 18 October 2000.

\section{Manisha Deshpande, Debashis Mitra, and Pradeep B. Parab National Centre for Cell Science Ganeshkhind, Pune, India}

